Hybrid intelligent parameter estimation based on grey case-based reasoning for laminar cooling process

Xing, Guishan and Ding, Jinliang and Chai, Tianyou and Afshar, Puya and Wang, Hong 2012 MIMS EPrint: 2012.15

Manchester Institute for Mathematical Sciences

School of Mathematics

The University of Manchester

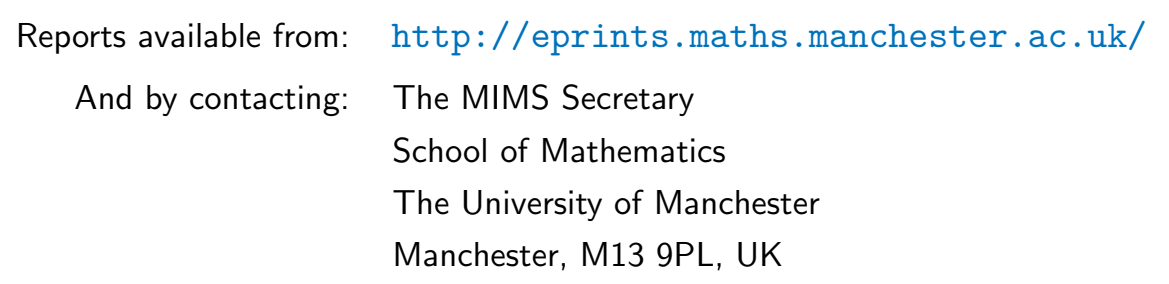




\title{
Hybrid intelligent parameter estimation based on grey case-based reasoning for laminar cooling process
}

\author{
Guishan Xing ${ }^{a}$, Jinliang Ding ${ }^{\mathrm{a}, *}$, Tianyou Chai ${ }^{\mathrm{a}}$, Puya Afshar ${ }^{\mathrm{b}}$, Hong Wang ${ }^{\mathrm{b}}$ \\ a State Key Laboratory of Integrated Automation for Process Industry, Northeastern University, Shenyang 110004, PR China \\ ${ }^{\mathrm{b}}$ Control System Centre, School of Electrical and Electronic Engineering, The University of Manchester, Manchester M13 9PL, UK
}

\section{A R T I C L E I N F O}

\section{Article history:}

Received 2 October 2010

Received in revised form

3 August 2011

Accepted 18 October 2011

Available online 16 November 2011

Keywords:

Laminar cooling process

Hybrid genetic algorithm

Grey case-based reasoning

Parameter estimation

\section{Introduction}

In steel manufacturing industry, the hot-rolled strip laminar cooling (HSLC) process is used to cool a strip from a finishing rolling temperature of roughly $820-920{ }^{\circ} \mathrm{C}$ down to a coiling temperature of $400-680^{\circ} \mathrm{C}$. Strips enter laminar cooling section after the finishing mill, then are cooled in the water cooling section, and finally are coiled by coiler. When the strip is cooled down on the run-out table, the mechanical properties of the corresponding strip are determined by the cooling curve (Tang et al., 2007; Sha et al., 2007). Therefore the highly flexible and precise control of the cooling curve and coiling temperature in the cooling section is extremely important for the product quality.

However, since it is difficult to measure the strip temperature continuously while in the cooling section, establishing a dynamic parametric model to estimate the strip temperature plays an important role in controlling the hot-rolled strip temperature. A fixedparameter model can represent a certain product type (i.e. grade). However, in strip manufacturing process, many different grades need to be produced which necessitates using a parameter-varying dynamic model to predict the temperature. Each grade is identified using a number of different process settings (e.g. moving speed, strip temperature, etc.). Then, in order to manufacture the required grade, the cooling water distribution is adjusted, so that the desired product

\footnotetext{
* Corresponding author. Fax: +862423895647.

E-mail addresses: jlding@mail.neu.edu.cn, jinliang.ding@gmail.com (J. Ding).
}

attributes are obtained. Consequently, rapidly changing operating conditions of cooling process lead to the instability of heat transfer characteristics. The model parameters represent the thermal characteristics of the cooling process, such as heat transfer coefficient which is influenced by many variables including ambient temperature, hardness grade of strip, strip thickness, temperature of cooling water, strip moving speed, strip temperature, and the distribution of cooling water. Since the resulting dynamic model parameters, e.g. the heat transfer coefficient are nonlinear and time varying, the mathematical model derivation is extremely difficult. Furthermore, traditional estimation techniques fail to estimate nonlinear parameters accurately due to the lack of prior process knowledge. In order to overcome these difficulties, an estimation technique based on casebased reasoning (CBR) has been considered in Tan and Chai (2005). However, the authors have considered to determine the weights of CBR features based on operator's expert experience, which incorporates significant uncertainties from one operator to another, and as a result, to inaccurate estimation of the parameters. In this paper, a new algorithm hybrid genetic algorithm (HGA) is developed to optimise the weights of CBR features.

\subsection{CBR background}

The CBR is formed of concepts and techniques which relate to knowledge representation, reasoning, and learning from experience (Aamodt and Plaza, 1994; Wettschereck and Aha, 1995; Bonzano et al., 1997; Jarmulak et al., 2000; Coyle and Cunningham, 2004; Xiong and Funk, 2006; Ahn et al., 2006; Sun and Li, 2009; 
Xiong, 2011). Perhaps the most significant advantage of CBR is similarity of its prediction approach to that of human reasoning system which makes predictive processes and results easily understandable to industrial users. It is known that human beings always search their memories to find similar experiences when they encounter a new problem. The human reasoning system can learn over time, reason in domains with incomplete or non-well-defined concepts, and provide means for explanation. The CBR is a well established methodology with broad applications such as medical science (Ahn and Kim, 2009), finance (Li and Ho, 2009), electronics (Chang et al., 2008), chemical engineering for quality design (Suh et al., 1998), intelligent Web-based sales service (Watson and Gardingen, 1999; Wilke et al., 1998), building and mechanical design (Nikolaychuk and Yurin, 2008; Rivard and Fenves, 2000; Mileman et al., 2002), material science (Amen and Vomacka, 2001; Mejasson et al., 2001), complex fault finding and troubleshooting (Aha et al., 1999) as well as planning and real-time scheduling tasks (Cunningham et al., 1997; Coello and dos Santos, 1999).

Generally, the process of CBR consists of four steps: retrieve, reuse, revise, and retain (Kolodner, 1993). To find a solution to a new problem case, it is first necessary to search similar cases. After assessing the similarity of the retrieved cases, the solution is reused. Alternatively, new knowledge is used to establish a new case, through the adaptation process. The performance of CBR relies on the composition of a case base, similarity assessment, and a case adaptation method, in which similarity assessment plays an important role (Aamodt and Plaza, 1994). Many CBR-based systems represent cases using features and employ a similarity function to measure the similarities between new and historical cases (Shin and Han, 1999). The approach commonly used to assess similarity is the distance function including the Euclidean distance (Cheng et al., 2008; Elhadi, 2000; Kwong and Tam, 2002) and the Manhattan distance (Bryant, 1997; Yu and Liu, 2006). Chiu et al. (2003) proposed GA-based feature weighting together with number of nonlinear similarity functions based on standard the Euclidean distance metric.

So far the main stream of the works involving similarity models has been focused on feature weighting (Kohavi et al., 1997; Wettschereck and Aha, 1995). Features are assigned with different weights in accordance with their importance and the global similarity metric is defined as a weighted sum of the local matching values in single attributes (Xiong, 2011). Different approaches have been proposed for estimating such weights automatically. Feature weights are modified by incremental learning according to success/ failure feedback of retrieval results (Bonzano et al., 1997; Ricci and Avesani, 1995). Probability-based techniques are used to assign weight values to features utilising conditional probabilities of classes and the probability of ranking principle (Creecy et al., 1992; Cercone et al., 1999). For weight adaptation, the case-ranking information is used as the similarity degree of case retrieval (Branting, 2001; Coyle and Cunningham, 2004; Stahl and Gabel, 2003). An accuracy improvement method has been proposed representing an approach for adapting the set of weights (Jarmulak et al., 2000; Ahn et al., 2006). Xiong (2011) has also proposed a new method to similarity assessment based on fuzzy rule-based reasoning and advocate that the set of fuzzy rules for similarity assessment can be learned from the case base using genetic algorithms.

\subsection{Hybrid genetic algorithm for optimisation}

GA has been widely applied to control systems and is one of the most powerful Artificial Intelligence (AI) techniques (Bedwani and Ismail, 2001). Compared with gradient-based search algorithms, GA is very suitable for optimisation problems with several local minima. It is also effective if the search space is either (partially) non-differentiable or discontinuous (Kargupta and Smith, 1991; Kristinsson and Dumont, 1992). The GA techniques have been applied to identify linear and non-linear systems by many researchers. For instance, Hossain et al. (1995) have used GA for parameter estimation of a flexible beam to design a vibration controller where a first order central finite difference (FD) method was used to study the behaviour of the beam.

The main disadvantages of GA, however, are the slow convergence to global optimum and the premature convergence. In order to overcome the convergence speed problem, this paper employs a truncation-based selection algorithm (Tavakolpour et al., 2010). The selection strategy involves replacing the weakest member of the present generation with the strongest member of the previous generation. This strategy improves the performance of the GA by ensuring monotonic improvement in the best fitness value of each generation. The second problem, i.e. premature convergence, is caused by the loss of diversity in population, especially when the search is continued for several generations (Gudla and Ganguli, 2005). Hence, an annealing algorithm is employed to overcome the drawback in the way that each member of a new population is obtained by the self-recognition crossover and mutation operators and then is determined whether to enter the next population according to the Metropolis criteria in annealing algorithm.

\subsection{Objectives and contributions}

The objective of this paper is to accurately estimate the cooling temperature model parameters so that the accuracy of predicting temperature distribution and cooling temperature of strip in the laminar cooling process are improved. For this purpose, a hybrid intelligent parameter estimation algorithm formed by a hybrid genetic algorithm and grey case-based reasoning is proposed. The HGA is used to optimise the weights of retrieval features in casebased reasoning which is considered an advantage over the existing methods in which the weight values are determined by expert experience. The weight vector is then used by grey casebased reasoning to accurately estimate the model parameters online. The proposed method is validated using a set of operational data gathered from hot-rolled strip laminar cooling process. Experiment results show significant precision improvement in the prediction of strip temperature in the laminar cooling process.

The rest of this paper is organised as follows. In Section 2, the laminar cooling process is presented, and then the problem of parameter estimation of the thermodynamic model is discussed. In Section 3, a detailed description of the proposed hybrid intelligent parameter estimation method is presented. Section 4 presents the implementation and results of the proposed method. Concluding remarks are made in Section 5.

\section{Process description and related work}

\subsection{Process description}

The schematic diagram of a laminar cooling process is illustrated in Fig. 1. Strips enter cooling section after the finishing mill at finishing rolling temperature of $820-920{ }^{\circ} \mathrm{C}$. After being cooled in the water cooling section, the strips are coiled at coiling temperature of $400-680^{\circ} \mathrm{C}$. Strips are $200-1100 \mathrm{~m}$ in length and $6.30-13.20 \mathrm{~mm}$ in thickness. There are 90 top headers and 90 bottom headers on the run-out table. The top headers that are of U-type are used for laminar cooling and the bottom headers that are of straight type are used for low pressure spraying. These cooling water headers are divided into 12 groups. The first nine groups are considered as the main cooling section and the last three groups are the fine cooling section. The corresponding thermodynamic model is presented in Appendix A. 


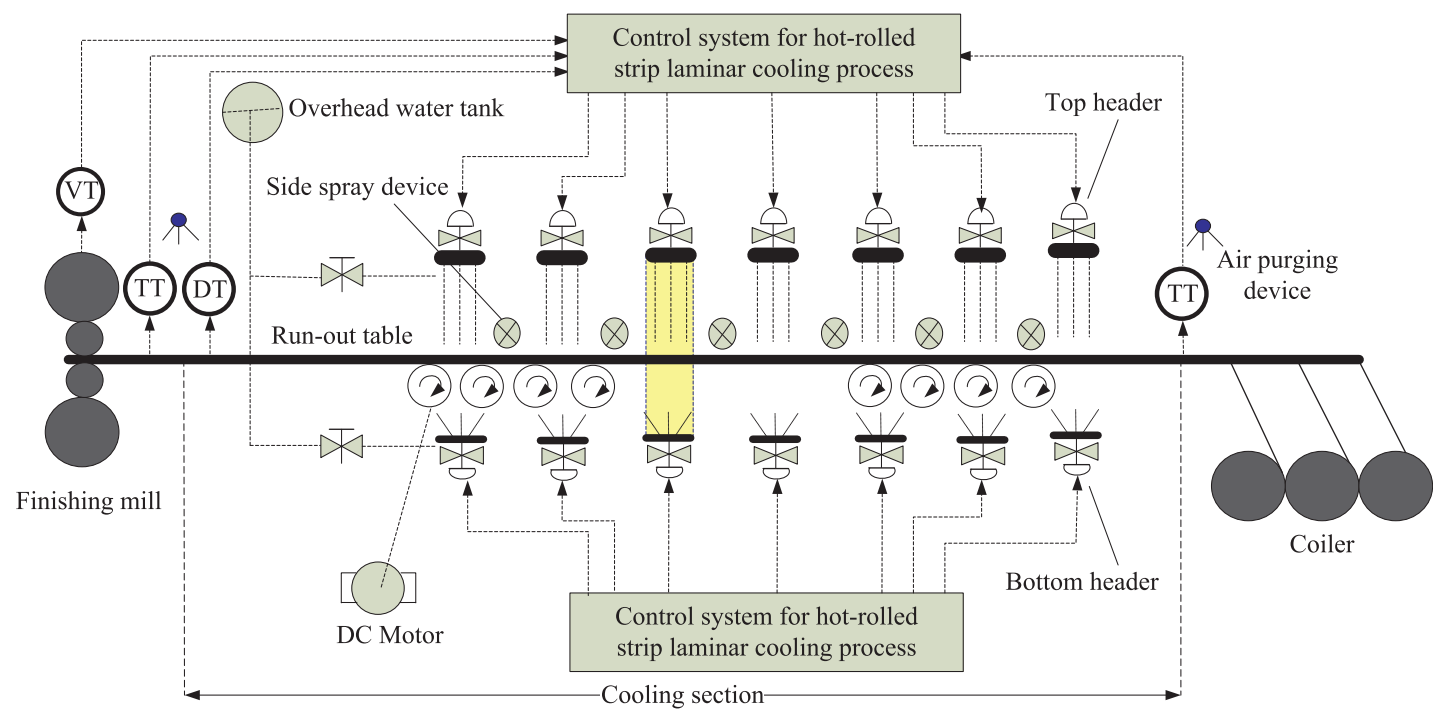

Fig. 1. Hot-rolled strip laminar cooling process. VT: pulse counter; TT: temperature transducer; DT: thickness transducer.

\subsection{Motivation for estimating model parameters}

The meaning of thermodynamic model parameters, $\alpha_{k}, \alpha_{v}, \alpha_{d}$, $\alpha_{T}, c$ and $\Delta v$, are fully explained in Appendix A, where

\section{$\alpha_{k} \quad$ proportional coefficient-influence on the accuracy of the dynamic model; \\ $\alpha_{v} \quad$ velocity parameter-represents the influence of finish rolling velocity increment on the heat transfer coefficient; \\ $\alpha_{d} \quad$ thickness parameter-represents the influence of finish rolling thickness increment on the heat transfer coefficient; temperature parameter-represents the influence of finish rolling temperature increment on the heat trans- fer coefficient; \\ $\Delta v \quad$ velocity increment-represents the influence of velocity increment on the thermal conductivity coefficients; \\ c velocity coefficient-represents the influence of velocity coefficient on the thermal conductivity coefficients.}

Since boundary conditions and strip temperature have nonlinear characteristics, functional relationship between the model parameters and the boundary conditions with input is nonlinear. Therefore, it is difficult to represent the model by mathematical first principle approach and therefore, an efficient estimation technique has to be applied. There is no prior knowledge concerning the functional relationship. Moreover, the model parameters are both time-varying and nonlinear. Therefore, traditional estimation methods are not appropriate. In this case, a hybrid intelligent parameter estimation algorithm based on grey case-based reasoning is used to estimate the parameters. The flow chart of the proposed estimation method is presented in Fig. 2. The estimated parameter values are used to calculate the model coefficients (i.e. the heat transfer coefficient, the thermal conductivity coefficient and the thermal diffusivity coefficient), and then the dynamic model is determined which can be used to calculate the temperature distribution of any point on the strip at any time. Hence, real-time temperature control for hot-rolled strip can be conducted.

\subsection{Related work}

Many models for laminar cooling process have been developed so far. However, the existing models are often not validated using practical data and remain as simulation models (Serajzadeh, 2004). Even in cases where the models are verified using industrial data, they assume fixed model parameters (Guo, 1997; Chai et al., 2002). However, neglecting the time-varying nature of the model parameters (i.e. heat transfer rate) would result in unstable heat exchange.

Therefore in this paper, the time-varying parameter estimation is the main focus in system identification of the laminar cooling process. The dynamic parameter tuning has been investigated so as to cope with the varying operating conditions in the laminar cooling process. The model parameters are determined by trial-anderror and back-calculating methods (Chai et al., 2002; Guo and Hwang, 1996), in which considerable human time besides computational effort is needed to select the model parameters. Moreover, the precision of the parameter estimation using these methods is not satisfactory. So the existing theoretical methods are difficult to apply in industrial practice. The nonlinear least-square and the nonlinear quadratic programming methods are well-developed techniques for parameter estimation (Fletcher and Xu, 1987; Tjoa and Biegler, 1991). However, if the search space is either (partially) nondifferentiable or discontinuous with respect to the parameters, the nonlinear least-square and the nonlinear quadratic programming methods often fail to determine the global optimum (Hossain et al., 1995). In addition, the laminar cooling is a complex process due to time-varying, highly nonlinear dynamics, non-existent mathematical representation of intermediate parameters, and also absence of online strip temperature measurement. As a result, the nonlinear least-square and nonlinear quadratic programming methods cannot be directly used in practice.

In this context, case-based reasoning (Tan and Chai, 2005) is a more suitable parameter estimation method, due to its speed and simplicity. However, the approach proposed in Tan and Chai (2005) has several drawbacks. First, the time-varying nature of the heat transfer coefficient is neglected. Therefore, since the control signal is calculated after parameter is determined, this might incorporate applying the control signal with wrong model parameters. Second, the weights of the case attributes in casebased reasoning are fixed by expert experience or by off-line experiments that cost time and effort. In addition, when historical case base has a significant change, since the weights of retrieval features are fixed by expert experience, the accurate estimate of parameters in the thermodynamic model cannot be realised.

An alternative approach is hybrid intelligent parameter identification based on Radial Basis Function (RBF) neural networks (Pian and Chai, 2008). In this approach, the time-varying behaviour of the heat transfer coefficient caused by the effect of the speed and the temperature changes is considered. However, 


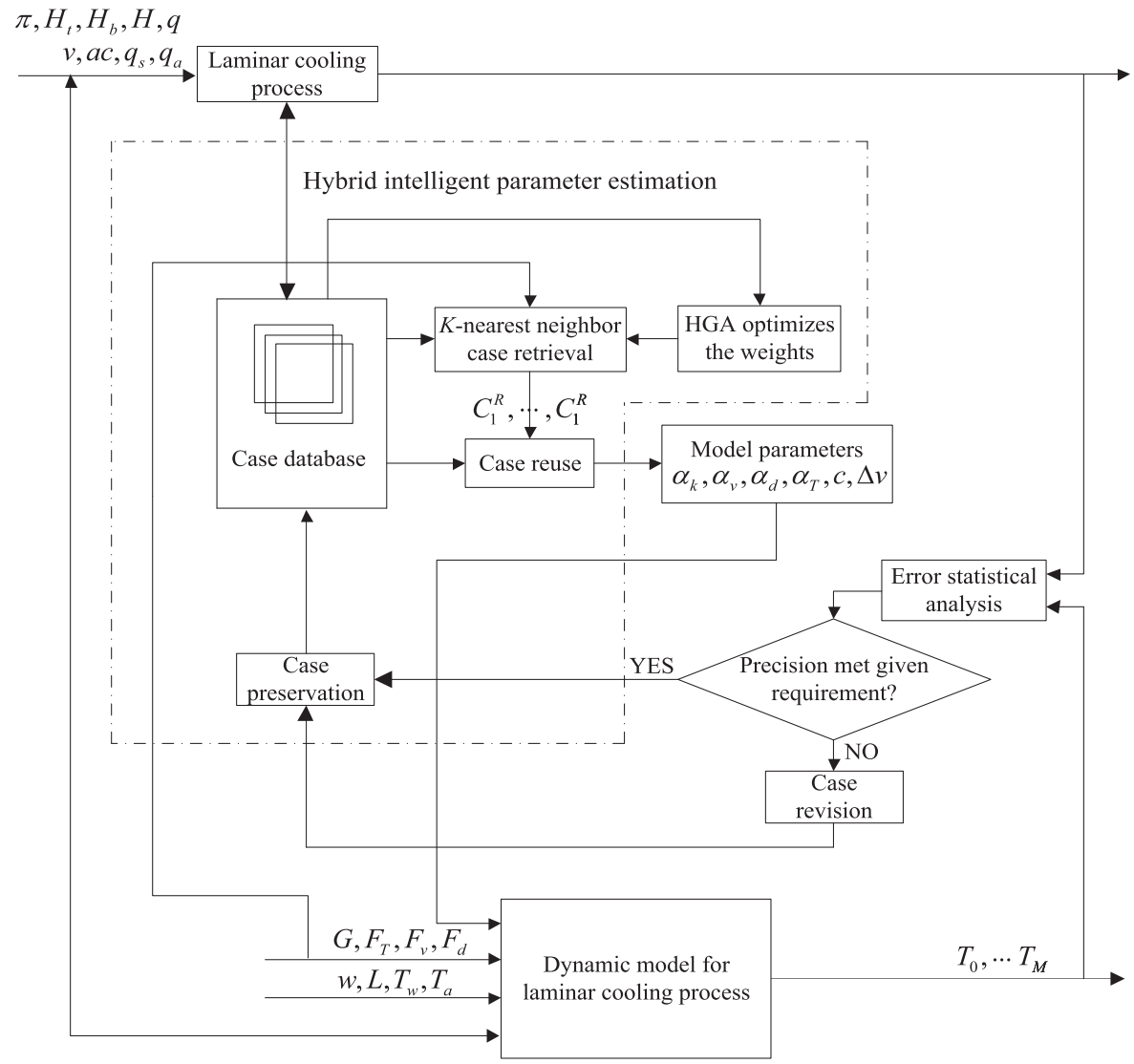

Fig. 2. Scheme of proposed hybrid intelligent parameter estimation algorithm.

the procedure to determine the weights using the RBF neural networks is time consuming. Hence, in a very small control period, this method is unable to complete the accurate estimation of the model parameters and the accurate estimate of strip coiling temperature cannot be completed as well.

\section{Hybrid intelligent parameter estimation based on grey case-based reasoning}

The basic idea of the proposed estimation method is presented in Fig. 2, which includes the grey case-based reasoning (CBR) and HGA. The weight vector of retrieval features in CBR is first optimised offline using HGA. Then the weight vector is used in the process of grey case-based reasoning.

\subsection{Grey case-based reasoning (GCBR)}

The boundary conditions and strip temperature have a nonlinear characteristic, which cause nonlinearity between the model parameters and the boundary conditions. In addition, the boundary conditions affecting the model parameters contain many qualitative and quantitative attributes, which are not necessarily known. In theory, there exists a grey relationship between various qualitative attributes and model parameters, which makes the grey decision method suitable for estimating the model parameters of laminar cooling process. Therefore, the case retrieval based on the grey similarity (Sun and Hui, 2006), which can address the grey relationship is adopted in the proposed GCBRbased hybrid intelligent parameter estimation algorithm for laminar cooling process.

\subsubsection{Case description}

The experiential knowledge of operating condition is expressed by a structured description in HSLC (Tan and Chai, 2005). Therefore the case representation based on frame structure is applied to represent the cases. The case in case base is composed of retrieval features and solution features. A series of qualitative or quantitative attributes are served as retrieval features of each case, which are denoted as $X=\left\{x_{1}, x_{2}, \cdots, x_{n}\right\}$. In this paper, the solution feature is quantitative attribute, which is denoted as $Y$. Namely, a case can be expressed as a tuple consisting of retrieval feature $X$ and solution feature $Y$. Thus, case model can be denoted as $u_{i}=$ Case $(X, Y)$. Case base composed of all cases can be denoted as $U=\left\{u_{i}\right\}$ $(i=1,2, \ldots, m)$, where $u_{i}$ represents a case.

\subsubsection{Establishment of case base}

In practice, since the model parameters, i.e. $\alpha_{k}, \alpha_{v}, \alpha_{d}, \alpha_{T}, c$ and $\Delta v$ (see Appendix A) cannot be measured online, we need to find the optimal parameters satisfying certain conditions to establish the case base. Since grid-search method can traverse variable space, it is inevitable for the optimal model parameters to be obtained by computing index function of each point in variable space. Moreover, equal step grid-search method is simple and feasible to determine the optimal model parameters $\alpha_{k}, \alpha_{v}, \alpha_{d}, \alpha_{T}$, $c, \Delta v$ by minimising the following performance index:

$J=\frac{1}{I} \sum_{i=1}^{I}\left|T_{c}^{i}-y^{i}\right|$

where $I$ is the number of strip segments. In fact, (1) indicates the mean absolute deviation between measured coiling temperature $T_{c}^{i}$ and the coiling temperature $y^{i}$ computed by the thermodynamic model of the top surface. Also in (1), $i$ indicates the $i$ th segment 
strip. The optimal values of the model parameters are computed by minimising $J$ in variable space. The resulting optimal parameters are then used alongside hardness grade $G$, strip thickness $F_{d}$, strip temperature $F_{T}$ and strip head velocity $F_{v}$ to form a case.

\subsubsection{K-nearest neighbour case retrieval based on the grey similarity}

In the present work, the retrieval strategy combining parameter optimisation method of hybrid genetic algorithm with $K$-nearest neighbour case retrieval is applied. There exists a kind of grey relation between various qualitative attributes and model parameters, which makes the traditional similarity difficult to deal with. However, it makes the grey similarity suitable for computing the similarity between two cases. Moreover, the calculation accuracy of grey similarity method is higher than the traditional similarity (Sun and Li, 2009). Therefore, grey correlation analysis (Sun and Hui, 2006) is applied for model parameter estimation.

The case base is expressed as $U=\left\{u_{i}\right\}(i=1,2, \ldots, m)$, and the set of retrieval features consist of practical operating conditions expressed as $X=\left\{x_{1}, x_{2}, \cdots, x_{n}\right\}$. Also, factor matrix consists of retrieval feature values of all cases denoted as $F$

$F=\left[\begin{array}{cccc}f_{11} & f_{12} & \cdots & f_{1 n} \\ f_{21} & f_{22} & \cdots & f_{2 n} \\ \vdots & \vdots & \ddots & \vdots \\ f_{m 1} & f_{m 2} & \cdots & f_{m n}\end{array}\right]=\left(f_{i j}\right)_{m \times n}$

where $f_{i j}$ is the value of the $i$ th case at $j$ th retrieval feature.

In the practical operating condition of HSLC, the retrieval features have different dimensions and orders of magnitude (e.g. strip hardness grade $G$, finishing rolling thickness $F_{d}$, finishing rolling temperature $F_{T}$ and finishing rolling velocity $F_{v}$ ). Therefore, it is necessary to normalise the primitive factor matrix to make the retrieval features with different dimension and order of magnitude comparable. Relative membership grade (RMG) of every retrieval feature is obtained as follows. The RMG matrix $F^{\prime}$ of retrieval features is obtained by normalisation. That is, each element is divided by the maximum of the corresponding column.

$F^{\prime}=\left[\begin{array}{cccc}f_{11}^{\prime} & f_{12}^{\prime} & \cdots & f_{1 n}^{\prime} \\ f_{21}^{\prime} & f_{22}^{\prime} & \cdots & f_{2 n}^{\prime} \\ \vdots & \vdots & \ddots & \vdots \\ f_{m 1}^{\prime} & f_{m 2}^{\prime} & \cdots & f_{m n}^{\prime}\end{array}\right]=\left(f_{i j}^{\prime}\right)_{m \times n}$

$f_{i j}^{\prime}=\frac{f_{i j}}{\max _{j} f_{i j}}$

where $\max _{j} f_{i j}$ is the maximum of $j$ th column.

It must be noted that the problem case is represented as $u_{0}$, and $f_{0 j}$ represents RMG obtained by normalising the $j$ th retrieval feature of $u_{0}$.

3.1.3.1. Grey relational degree of attribute. According to formulation of grey relational degree, the relational degree $\gamma_{i j}$ between case $u_{0}$ and case $u_{i}$ at retrieval feature is defined as

$\gamma_{i j}=\frac{\min _{j}\left|f_{0 j}^{\prime}-f_{i j}^{\prime}\right|+b \max _{j}\left|f_{0 j}^{\prime}-f_{i j}^{\prime}\right|}{\left|f_{0 j}^{\prime}-f_{i j}^{\prime}\right|+b \max _{j}\left|f_{0 j}^{\prime}-f_{i j}^{\prime}\right|}$

where $\max _{j}\left|f_{0 j}^{\prime}-f_{i j}^{\prime}\right|$ and $\min _{j}\left|f_{0 j}^{\prime}-f_{i j}^{\prime}\right|$ are the maximum and minimum of membership grade distance between the problem case and every case in $U$ at $j$ th retrieval feature, respectively. Furthermore, $\left|f_{0 j}^{\prime}-f_{i j}^{\prime}\right|$ is the membership grade distance between the problem case and case $u_{i}$ in $U$ at $j$ th retrieval feature. $b$ is environment parameter, and $b \in[0,1]$. Obviously, $\gamma_{i j} \in[0,1]$ so that the grey relational degree matrix between the problem case and the case in case base is $R=\left\{\gamma_{i j}\right\}$ with the dimension $m \times n$.

Similarity calculation method based on distance is employed in this paper. Grey similarity $\operatorname{sim}_{0 i}$ (Sun and $\mathrm{Li}, 2009$ ) between problem case and historical case $u_{i}$ is defined as

$\operatorname{sim}_{0 i}=1-\left\{\sum_{j=1}^{n}\left[\omega_{j}\left(1-\gamma_{i j}\right)\right]^{h}\right\}^{1 / h}$

where $h$ is a distance parameter, which is the Manhattan distance when $h=1$ and the Euclidean distance when $h=2$. Also, $\omega_{j}$ is the weight of $i$ th retrieval feature. The weights of $n$ retrieval features form feature weight vector $W=\left\{\omega_{1}, \omega_{2}, \cdots, \omega_{n}\right\}$, the value of which is optimised by the proposed HGA. Greater the value of $\operatorname{sim}_{0 i}$, greater the similarity between problem case $u_{0}$ and historical case $u_{i}$. In the present work, the Euclidean distance (i.e. $h=2$ ) is applied.

3.1.3.2. K-nearest neighbour case retrieval. Similarity threshold $T$ is set according to $k$-nearest neighbour. $K$-nearest neighbour case set $U_{*}$ of the problem case $u_{0}$ is composed of all historical cases whose similarities are equal to or greater than $T$ and $U^{*}$ is defined as

$U^{*}=\left\{u_{i^{*}} \mid \operatorname{sim}_{0 i^{*}} \geq T\right\}\left(i^{*}=1,2, \cdots, L\right)$

The value of the threshold $T$ can be calculated using

$T=\rho \max \left(\operatorname{sim}_{0 i}\right)$

where $\max \left(\operatorname{sim}_{0 i}\right)$ is the maximum of similarity and $\rho$ is the percentage.

In cases where the sub-historical case base in the historical case base have the same hardness grade and thickness (main indices) with the problem case, the weight vector of the problem case is known. That is, one of the sub-historical case bases and then the case retrieval can be conducted. When there are no cases in the historical case base having the same hardness grade and thickness (main indices) with the new problem case, the weight vector of the new problem case is unknown, which makes the case retrieval impossible. Thus, in the latter cases, the following calculation procedure is carried out.

First, establish the case structure of the weight vector $W=\left\{\omega_{1}, \omega_{2}, \cdots, \omega_{n}\right\}$, in which the retrieval features are $G, F_{d}, F_{T}$, $F_{v}$ and the solution is the weight vector $W$. The case structure of the weight vector $W$ is shown in Table 1 .

Then, the retrieval features of the sub-historical case base and the weight values of retrieval features corresponding to the subhistorical case base form a case. In this way, the case base of the weights can be established. When a new type of steel (a new problem case) is required to be cooled, which has never been produced in a steel industry, the weight vector of the new problem case is obtained using CBR for the case base of the weight vector.

With the weight vector of the new problem case obtained by above method, the whole historical case base serves as the retrieval case base and the model parameter values corresponding to the new problem case can be obtained by CBR. Since there are no similar

Table 1

Case structure of the weight vector $W$.

\begin{tabular}{lllll}
\hline \multicolumn{2}{l}{ Retrieval feature } & & & Solution \\
\hline$x_{1}$ & $x_{2}$ & $x_{3}$ & $x_{4}$ & $Y$ \\
$G$ & $F_{d}$ & $F_{T}$ & $F_{v}$ & $W$ \\
\hline
\end{tabular}


cases in case base for a new type of steel, the resulting model parameter values are only provisional model parameter values.

\subsubsection{Case re-use based on replacement method}

Using the similarities between the problem case and retrieved cases as weight values, the solution of the problem case can be obtained with the weighed sum of all retrieved similar cases. Greater the similarity between the problem case and retrieved case, greater the weight value of the corresponding case solution. In other words, the corresponding case solution is in large proportion in the solution of the problem case. Once the re-use solution is obtained, it is used for appropriate case solution.

\subsection{Local search method}

Since the fitness function is not differentiable, we have to use methods that do not use gradients. Therefore the multidimensional minimisation method based on inverse parabolic interpolation was employed as a Local Search (LS) method in the Hybrid Genetic Algorithm (HGA).

Almost all multidimensional minimisation methods use onedimensional minimisation (Makeyev et al., 2010). One-dimensional minimisation methods include inverse parabolic interpolation, golden section search, and their combinations (e.g. Brent's method Press et al., 2007). Here it is assumed that GA stops at the solution nearest to the global extreme of the fitness function and that in each direction in the neighbourhood of the global extreme of the fitness function is approximately parabolic. The smoothness of the fitness function suggests that in the neighbourhood of the global extreme the first three terms of Taylor's series can approximate the fitness function. Indeed, it is approximately parabolic in this neighbourhood. Then the abscissa value corresponding to the extreme of the parabola which can be fitted through any three different points should lie at least very close to the abscissa value of the global minimum (Makeyev et al., 2010). Thus, the inverse parabolic interpolation should be suitable under given assumptions in this research.

\subsection{Hybrid genetic algorithm}

Since hardness grade and thickness have a greater influence on model parameters, they can be used as main indices in case retrieval, while speed and temperature which have a less influence on model parameters can be used for secondary indices. Historical case base can be sub-divided into sub-historical case bases according to the main indices. In each sub-historical case base, the hardness grade and thickness of all the cases are same. The weight values of retrieval features corresponding to the subhistorical case base are calculated by the hybrid genetic algorithm. When the problem case is formed, a search is carried out in the historical case base to retrieve the sub-historical case base with the same hardness grade and thickness as the problem case. In the next step, the search is performed within the sub-historical case base retrieved from the first step above. The goal for this search is to obtain the solution of the problem case. If the subhistorical case base having the same hardness grade and thickness with the problem case is not found by searching, the whole case base is served as the retrieval case base and the weight values of retrieval features are calculated by the hybrid genetic algorithm. Then the solution of the problem case can be retrieved using the optimised weight vector. The retrieval accuracy can be improved by above mentioned method. When the historical case base changes significantly (equivalent to an increase of more than $30 \%$ over the number of cases) or a case having new hardness grade and thickness joins the historical case base, the weight vector of retrieval features corresponding to sub-historical case base having significant change and new type of steel should be recounted to improve the retrieval accuracy of problem case solution. The drawback that the weight values of retrieval features are fixed through engineering experience can be overcome by the above process. When the case base changes significantly, recounting of retrieval feature weight vector can improve the retrieval accuracy using hybrid genetic algorithm.

The GA can converge to a near-optimal solution quickly, but the accuracy in reaching the optimum solution is poor. Local search algorithms are likely to stop at a solution which is the closest solution to the starting point. It would reach a solution which is very close to optimal solution if the local search method starts near the global optimum.

In this paper, a multidimensional minimisation method, which is based on the inverse parabolic interpolation (Goldberg, 1989) is used as a local search method. The LS method is applied for post-hybridisation of GA. The flow chart of the proposed HGA is presented in Fig. 3.

The proposed HGA is performed to realise the parameter optimisation in the following two consecutive steps. Firstly, in order to find an area with low fitness values in the search space, GA algorithm is executed and then the local search method is applied to the best set of weights found by GA. In contrast to LS method, a precise initial guess is not required for such HGA which can find good solutions faster than the standard GA. To overcome the drawback of genetic algorithm and improve the local search performance of GA, diversity of evolution population should be enhanced. The optimum can be found in the neighbourhood of optimal solution with low fitness values and the optimum can be found as well in the neighbourhood of poor solution with high fitness values. Accordingly, in order to improve the local search performance of GA, an annealing algorithm is adopted in this paper. Initial population is optimised by the genetic operations of GA, namely, crossover and mutation are performed on the initial population at self-recognition crossover and mutation probabilities to obtain a new population. Each member is determined whether to enter the next population according to Metropolis criteria in the annealing algorithm and GA proceeds using the population as parental generation (Liu et al., 2009). The whole process is repeated until the termination conditions meet.

\subsubsection{Generation of initial population}

It has been known that real-coded GAs are more suitable for large dimensional search spaces than binary-coded GAs since they are more precise, consistent and they lead to faster convergence (Baskar et al., 2003, 2004). Moreover, a real-parameter GA does not require the knowledge of bounds on parameter values and can be applied in problems where such information is not available (Subbaraj et al., 2011). Thus real-coded GA is employed in this paper.

In HGA, each chromosome is encoded as a vector of floatingpoint numbers, with the same length as the vector of weights in GCBR. For convenience, $\left[\omega_{1}, \omega_{2}, \ldots, \omega_{i}, \ldots, \omega_{N}\right]$ is represented as a vector of chromosomes to the solution of the weight vector in case retrieval of GCBR. Initialisation of $M$ individual population is carried out using

$\omega_{i}=\omega_{i}^{l}+\sigma_{i}\left(\omega_{i}^{u}-\omega_{i}^{l}\right)$

where $\omega_{i}^{u}$ and $\omega_{i}^{l}$ are the domain of $\omega_{i}$, where $\omega_{i}^{u}=1$ and $\omega_{i}^{l}=0$. Also, $\sigma_{i}$ is a random number uniformly distributed over $[0,1]$. The tuning law (9) is repeated $N$ times to produce the vector $\left[\omega_{1}, \omega_{2}, \ldots, \omega_{i}, \ldots, \omega_{N}\right]$. By repeating the above procedure $M$ times, $M$ uniformly distributed initial feasible solutions in the search space are obtained. 




Fig. 3. Flow chart of proposed hybrid genetic algorithm (HGA).

\subsubsection{Fitness function of $G A$}

The estimation error $e_{i}$ between the optimal value $R_{i}$ and the estimated value $Y_{i}$ of model parameter is

$e_{i}=R_{i}-Y_{i}$

The mean square error $\varepsilon$ is defined as

$\varepsilon=\frac{1}{N} \sum_{i=1}^{N}\left(R_{i}-Y_{i}\right)^{2}$

where $N$ is the number of cases in historical case base.

An obvious approach is then to estimate the parameters of strip coiling temperature model so as to fit the estimated value $Y_{i}$ as closely as possible to the optimal value $R_{i}$ as determined in Section 3.1.2. In fact, the parameters of strip coiling temperature model should be estimated so that the mean square error $(\varepsilon)$ of the estimated parameter converges to zero. Therefore, the mean square error $(\varepsilon)$ of the estimated parameter is used as fitness function of GA.

\subsubsection{Selection strategy}

In this study, the selection strategy as in Tavakolpour et al. (2010) is applied for the GA's selection criteria. Since parameter estimation is a minimisation problem, it is natural to assume that the weakest feasible solution is the one which has the highest mean square errors. At the other extreme end, the strongest individuals are associated with smallest values of mean square error. The individuals with smaller mean square error values will replace those with higher mean square error values. Consequently, the new population is found by the selection operator so that the mean square error of the fitness function is minimised. The probability values, which are used in the selection strategy are determined through trial-and-error to find the best performance.

\subsubsection{Self-recognition crossover and mutation}

Premature convergence which is the main disadvantage of GA is caused by the loss of diversity in population, especially when the search is continued for several generations (Gudla and Ganguli, 2005). It has been shown by Liu et al. (2009) that the self-recognition crossover and mutation operators have the capability to overcome the mentioned drawback, which is employed in this paper. Crossover probability $p_{c}$ and mutation probability $p_{m}$ change with fitness values. That is, the individuals with higher fitness (mean square error) are associated with greater crossover and mutation probability values. When the GA is stuck in a local optima, the individuals with smaller fitness (mean square error) are associated with greater crossover and mutation probability values as well, which can contribute to inhibit the premature convergence of GA.

\section{Implementation and results}

\subsection{Training}

In this section, the experiment results conducted on a laminar cooling process are presented. In this example, the number of the thickness grade range of low-carbon steel produced by a steel plant is 500. Thickness range of strip cooled by laminar cooling system is $1.1-28 \mathrm{~mm}$, which is divided into 15 grades. In this study, 179 strip data (8117 sampling points) are used for experiment, in which the thickness grade is 9 (i.e. the thickness is between 
$10.6 \mathrm{~mm}$ and $12.6 \mathrm{~mm}$ ). The temperature modelling is conducted on 11 points along the thickness direction. Also, 139 strip data (6469 sampling points) are used to optimise the weight values of retrieval features offline and to establish the initial case base. Furthermore, 40 strip data (1648 sampling points) are used to illustrate the validity of the proposed method. Since the temperature of strip on the head and the tail varies with changing operating conditions, in order to guarantee the generality of the experiment, the middle segment of every strip is used as test case for the experiment.

The estimated value of the parameters $\alpha_{k}, \alpha_{v}, \alpha_{d}, \alpha_{T}, c$ and $\Delta v$ are used to validate the proposed method. It is worth mentioning that estimation of $\alpha_{k}$ is continued within each control period and the rest are estimated before the control starts.

The estimation strategy explained earlier is implemented within MATLAB $^{\mathrm{TM}}$ environment. The goal is to evaluate the effectiveness of proposed method and compare it against existing estimation methods. The proposed algorithm yields the model parameter weights summarised in Table 2 .

Fig. 4. shows the convergence of the HGA. The minimum mean square error of 10.29 was determined after 70 generations. The value of parameter $\alpha_{k}$ estimated by the proposed method is illustrated in Fig. 5. It is noted that the proposed method has effectively estimated the model parameters. Fig. 6 represents the error variations between the estimated values using the proposed method and the optimal value from the simulation.

In this research a new parameter estimation method was proposed for the estimation of the model parameters. Table 3 summarises the HGA parameters employed in the estimation scheme.

\subsection{Validation}

Once a parameter estimation method is proposed, it is required to validate whether the method is good enough to estimate the parameters accurately. There are two ways to investigate the validity of an estimation method (Soderstrom and Stoica, 1984).

- Using plots, visual exploration, and experience;

- Performing statistical tests on the prediction error.

While visual exploration of the predictions and comparing them against the measured data provide convenient means for model validation, statistical residual analysis (e.g. independence or whiteness tests) provides more concrete means to assess the performance of an estimation algorithm.

\subsubsection{Plots and visual exploration}

In order to evaluate the proposed method, different types of strips should be employed. As in this paper, four strips of different types are employed for the experiment. Then the results are compared with the measured coiling temperature of the strip points.

Fig. 7 reveals the method capability to predict the coiling temperature for different types of strips. As can be seen, the

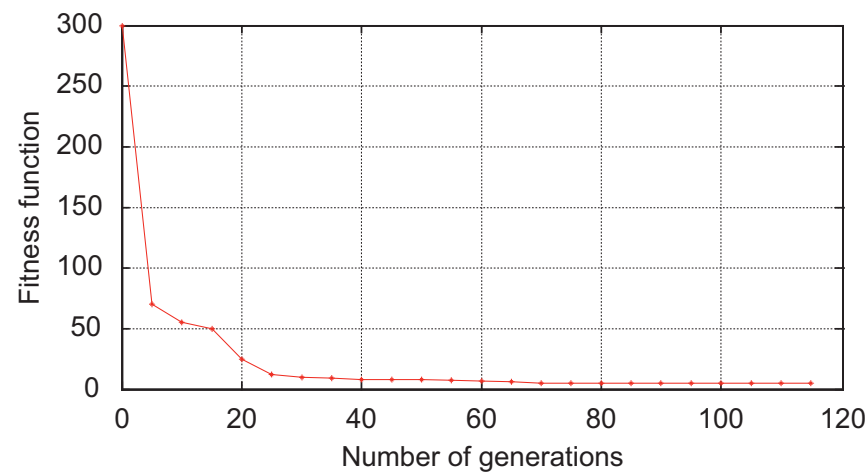

Fig. 4. Convergence of the HGA.

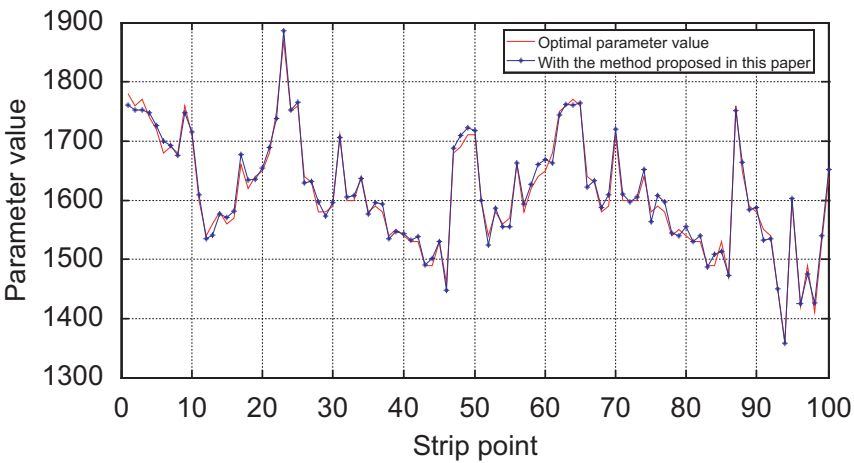

Fig. 5. Estimation of parameter $\alpha_{k}$ using the proposed method.

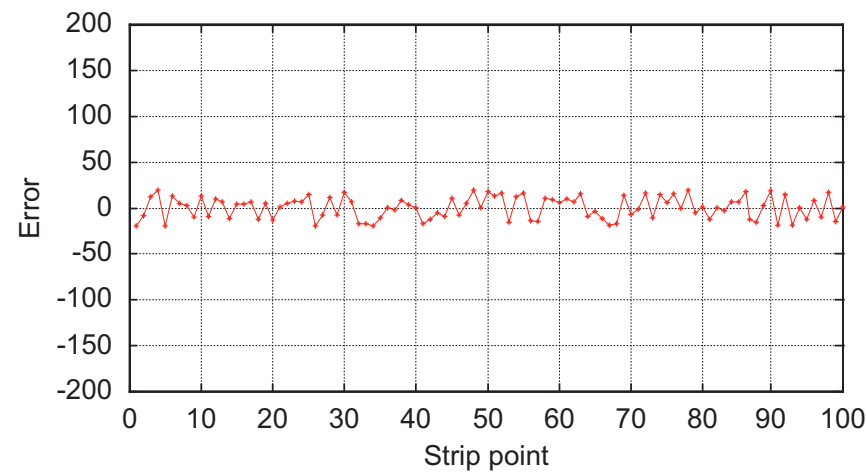

Fig. 6. Error between optimal and estimated values of the parameter $\alpha_{k}$.

Table 2

Estimated weights of model parameters via HGA.

\begin{tabular}{|c|c|c|c|c|c|c|}
\hline \multirow{2}{*}{$\begin{array}{l}\text { Parameter } \\
\text { Weight }\end{array}$} & \multicolumn{4}{|l|}{$\alpha_{k 0}$} & \multicolumn{2}{|l|}{$\Delta \alpha_{k}$} \\
\hline & $\omega_{1}(G)$ & $\omega_{2}(d)$ & $\omega_{3}(T)$ & $\omega_{4}(v)$ & $\omega_{1}\left(\Delta T_{0}\right)$ & $\omega_{2}(\Delta v)$ \\
\hline Value & 0.63 & 0.51 & 0.45 & 0.26 & 0.57 & 0.43 \\
\hline Parameter & $c$ & & & $\Delta v$ & $\alpha_{v}$ & $\alpha_{T}$ \\
\hline Weight & $\omega_{1}(G)$ & $\omega_{2}(T)$ & $\omega_{3}(d)$ & $\omega(v)$ & $\omega(v)$ & $\omega(T)$ \\
\hline Value & 0.67 & 0.12 & 0.39 & 0.59 & 0.74 & 0.23 \\
\hline
\end{tabular}


Table 3

The HGA parameters.

\begin{tabular}{ll}
\hline Parameters & Type/value \\
\hline Population size & 120 \\
Parameter range & {$[0,1]$} \\
Selection strategy & Truncation-based selection \\
Mutation & Uniform \\
Mutation probability & 0.01 \\
Crossover & Double points \\
Crossover probability & 0.6 \\
LS method & Inverse parabolic minimisation \\
Number of runs of LS method & 10 \\
\hline
\end{tabular}

proposed method has predicted the coiling temperature of different types of strips accurately in Fig. 7(a)-(d).

\subsubsection{Statistical tests}

It is known that the auto-correlation and cross-correlation tests are the most useful statistical methods for model validation (Tavakolpour et al., 2010). The auto-correlation and cross-correlation tests are carried out to determine the effectiveness of the proposed method. The results of the auto-correlation and crosscorrelation tests are shown in Fig. 8(a) and (b), respectively.

\subsection{Comparison and analysis}

A question may arise about the performance of the proposed method compared to other existing estimation methods. To address this, a comparative study was carried out between the existing methods and the proposed method. The existing methods (Tan and Chai, 2005; Pian and Chai, 2008) and the proposed method were executed 10 times. The maximum of generations was restricted to 120 . The estimation scheme was run until the mean square error became less than 10.5.

Table 4 shows the comparative results obtained from the proposed method and the existing methods. It can be clearly seen that the proposed method has estimated the model parameters more effectively than the existing methods. The method addressed in (Tan and Chai, 2005) has not achieved the desirable mean square error of 10.5 , which may be attributed to the drawback that the weight values are fixed by the expert experience. Fig. 9 shows the estimation error of the parameter $\alpha_{k}$ using the existing methods and the proposed method. The RMS error and maximum absolute error of the three methods in the estimation of parameter $\alpha_{k}$ are shown in Table 4. As shown in Fig. 9 and Table 4, it is can be seen that the proposed method contributes significant accuracy improvement in the parameter estimation. Moreover, it is fast and effective and may be used in real-time control of strip temperature in the laminar cooling process.

The RMS error and maximum absolute error are defined as follows:

$R M S E=\sqrt{\frac{1}{I} \sum_{i=1}^{I}\left[R_{i}-Y_{i}\right]^{2}}$

$M A X E=\max \left\{\left\|R_{1}-Y_{1}\right\|,\left\|R_{2}-Y_{2}\right\|, \cdots,\left\|R_{I}-Y_{I}\right\|\right\}$

where I stands for the number of the cases in test case base.

In order to evaluate the effectiveness of the proposed method, 196 samples of four strips under different operating conditions are used. Fig. 10 shows the comparison of the estimation error of the coiling temperature among the existing methods and the proposed one. The forecasting results of coiling temperature using the three methods are shown in Table 5. As shown in Fig. 10 and
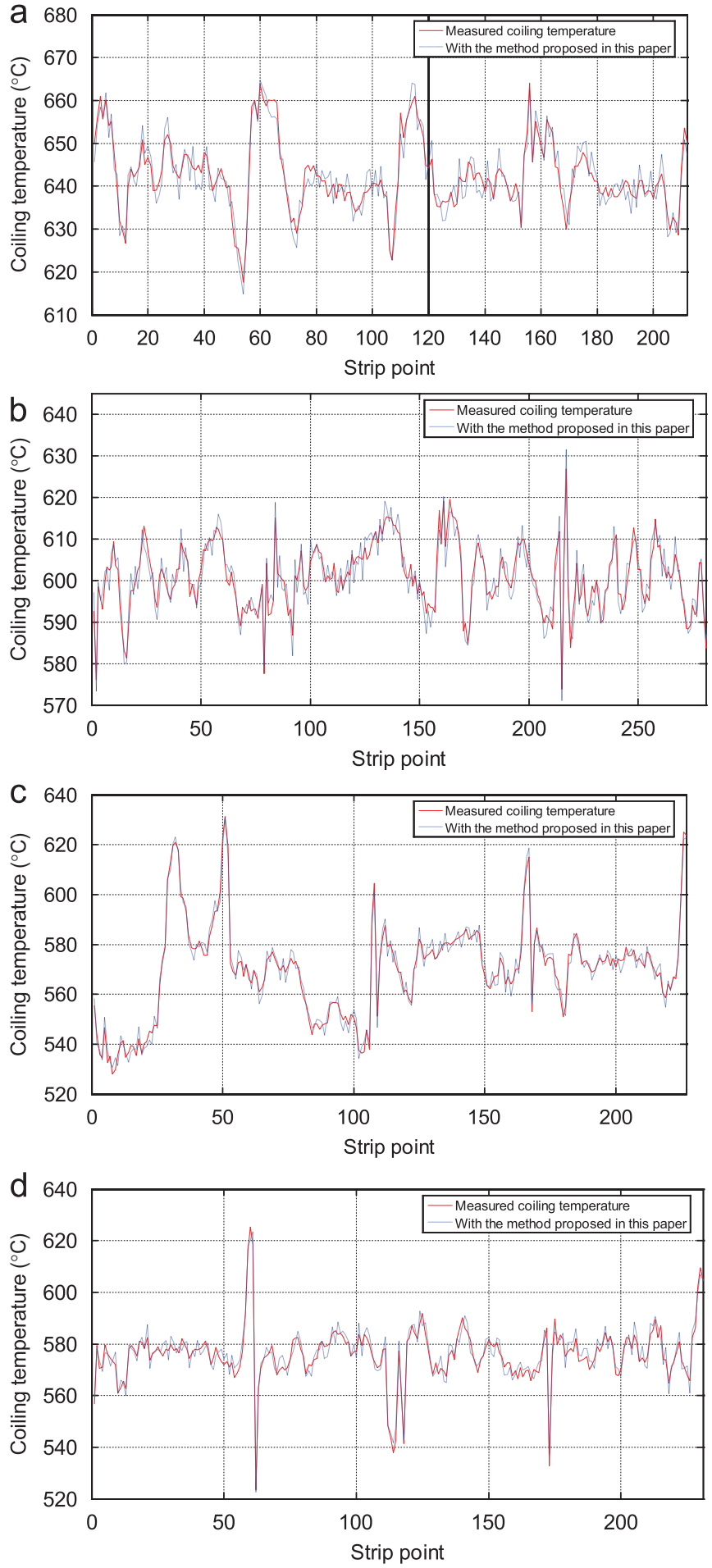

Fig. 7. Predicting precision of coiling temperature to different types of strips Hardness grades: (a) 195; (b) 226; (c) 192; and (d) 501.

Table 5, it can be seen that the average error is 17.28 and $10.53{ }^{\circ} \mathrm{C}$ in Tan and Chai (2005) and Pian and Chai (2008), respectively, and 118 and 154 segments are controlled in the range of $\pm 20{ }^{\circ} \mathrm{C}$ with hit rate of $60.2 \%$ and $78.6 \%$, respectively. However, with the proposed method, the average prediction error is $4.69{ }^{\circ} \mathrm{C}$ and the coiling temperatures of 190 segments are controlled in the range of $\pm 5{ }^{\circ} \mathrm{C}$ with the hit rate of $96.9 \%$. Therefore, experiment results show that the proposed method is effective in the parameter estimation. 


\section{Conclusions}

A hybrid intelligent method based on grey case-based reasoning is proposed to estimate the parameters of strip thermodynamic model in HSLC. The problem is formulated as a non-linear optimisation problem. To verify the feasibility, the proposed method was tested on a set of industrial data from HSLC and the results were compared with the existing parameter estimation methods (Tan and Chai, 2005; Pian and Chai, 2008). The proposed method has been found to be more accurate as it gives minimum RMSE and MAXE than the existing methods. By analysing the results, it can be observed that the proposed parameter estimation method gives better results than other methods. The future work will be focused on the practical application study of the proposed method. Moreover, the computational burden of the weight vectors
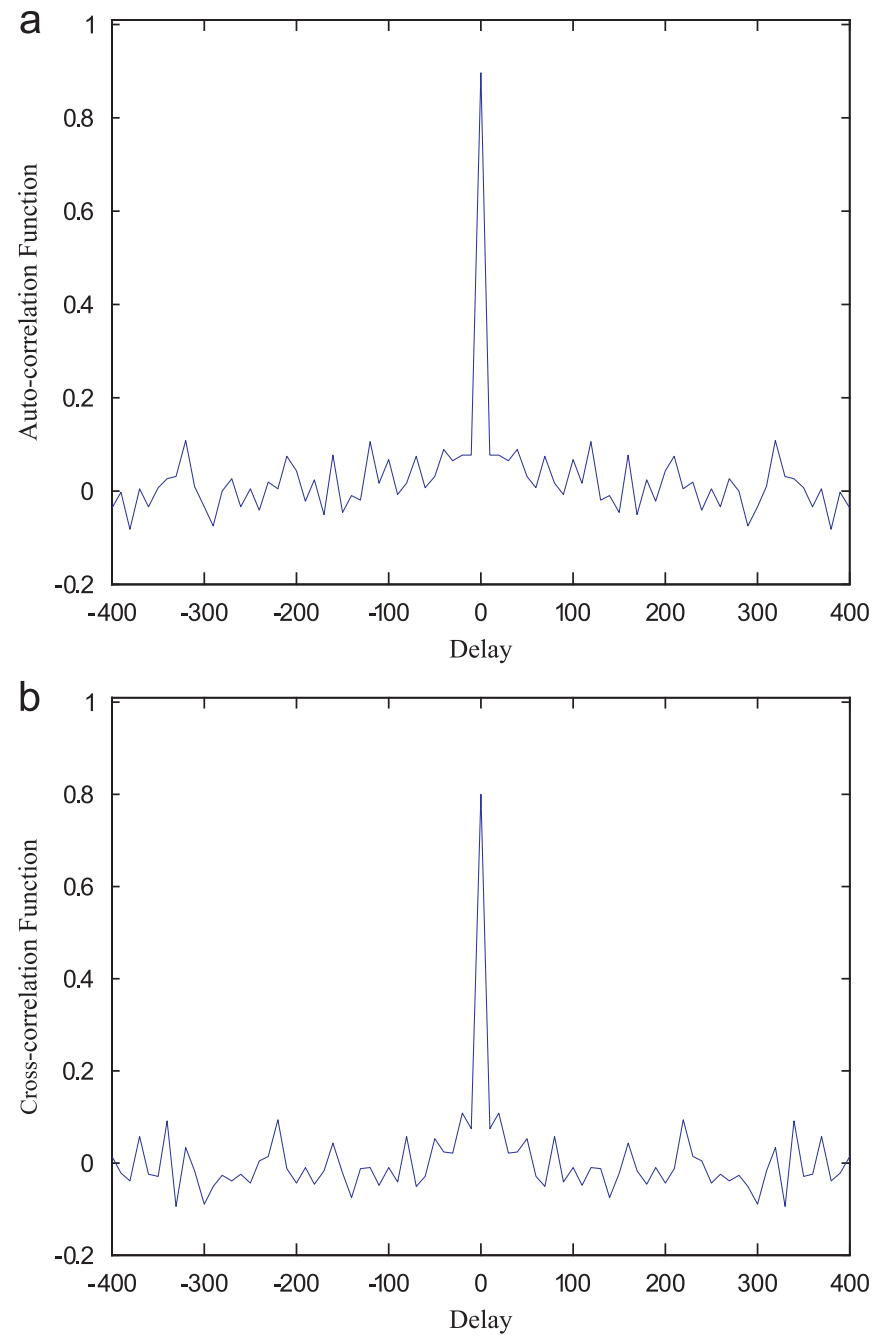

Fig. 8. Correlation tests of the proposed method. (a) Auto-correlation of the residuals and (b) cross-correlation. of retrieval features in grey case-based reasoning optimised using the proposed hybrid genetic algorithm offline is considerable. Therefore, the fast hybrid genetic algorithm is still a problem to be solved in future work.

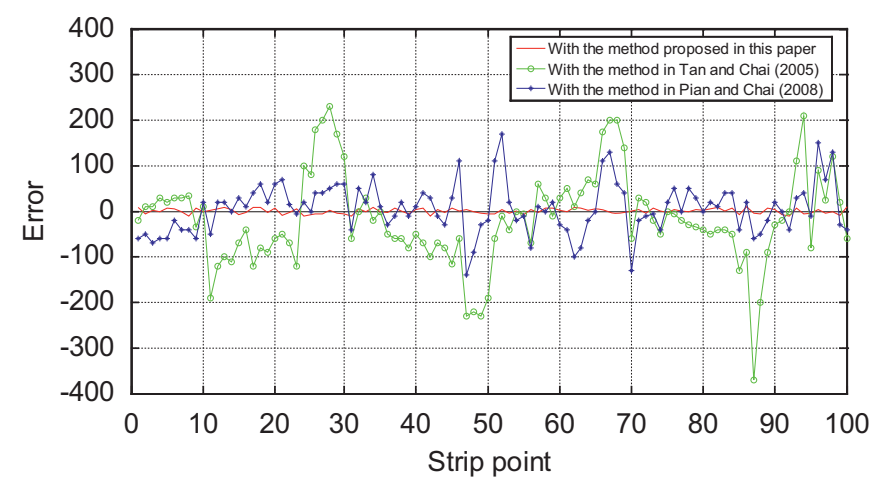

Fig. 9. Comparison of the estimation precision of parameter $\alpha_{k}$ among the three methods.



Fig. 10. Comparison of forecasting precision of coiling temperature among the three methods.

Table 5

Forecasting results of coiling temperature.

\begin{tabular}{|c|c|c|c|}
\hline Adopted method & $\begin{array}{l}\text { Method of Tan } \\
\text { and Chai (2005) }\end{array}$ & $\begin{array}{l}\text { Method of Pian } \\
\text { and Chai (2008) }\end{array}$ & $\begin{array}{l}\text { The proposed } \\
\text { method }\end{array}$ \\
\hline Number of segments & 196 & 196 & 196 \\
\hline Mean forecasting error & 17.28 & 10.53 & 4.69 \\
\hline RMSE & 15.36 & 10.80 & 4.21 \\
\hline MAXE & 32.25 & 25.14 & 6.58 \\
\hline $\begin{array}{l}\text { Number of segments } \\
\left(\Delta T \text { in } \pm 30^{\circ} \mathrm{C}\right)\end{array}$ & 136 & 171 & 196 \\
\hline $\begin{array}{l}\text { Number of segments } \\
\left(\Delta T \text { in } \pm 20^{\circ} \mathrm{C}\right)\end{array}$ & 118 & 154 & 196 \\
\hline $\begin{array}{l}\text { Number of segments } \\
\left(\Delta T \text { in } \pm 5^{\circ} \mathrm{C}\right)\end{array}$ & 3 & 5 & 190 \\
\hline
\end{tabular}

Table 4

The performance comparison of in parameter estimation of $\alpha_{k}$ among the proposed method and the existing methods (Tan and Chai, 2005; Pian and Chai, 2008).

\begin{tabular}{|c|c|c|c|c|c|c|}
\hline \multicolumn{2}{|c|}{$\begin{array}{l}\text { Parameter estimation with the } \\
\text { method (Tan and Chai, 2005) }\end{array}$} & \multicolumn{2}{|c|}{$\begin{array}{l}\text { Parameter estimation with the } \\
\text { method (Pian and Chai, 2008) }\end{array}$} & \multicolumn{2}{|c|}{$\begin{array}{l}\text { Parameter estimation With } \\
\text { the proposed method }\end{array}$} & \multirow{2}{*}{$\begin{array}{l}\text { RMSE rate of improvement (\%) The proposed } \\
\text { method vs. the methods (Tan and Chai, 2005; } \\
\text { Pian and Chai, 2008) }\end{array}$} \\
\hline RMSE & MAXE & RMSE & MAXE & RMSE & MAXE & \\
\hline 72.58 & 163.25 & 53.81 & 112.41 & 10.29 & 21.83 & $41.5 \%, 25.4 \%$ \\
\hline
\end{tabular}




\section{Acknowledgements}

This work was supported in part by the Chinese National Fundamental Research Program (2009CB320601), the NSF of China (61020106003, 60821063 and 60904079), the 111 project (B08015), CICADA under Grant EP/E050441/1 and the U.K. Leverhulme Trust (F/00 120/BC).

\section{Appendix A}

Using temperature-dependent thermal property (Pehlke et al., 1982), the latent heat is considered and the thermodynamic model (Zheng et al., 2009) is presented as

$\dot{T}=\frac{-\lambda}{\rho c_{p}} \frac{\partial^{2} T}{\partial z^{2}}-\dot{l} \frac{\partial T}{\partial l}$

with the boundary conditions on its top and bottom surfaces

$\pm \lambda \frac{\partial T}{\partial z}=h\left(T-T_{\infty}\right)$

where the right hand side of above formula stands for $h$ times $\left(T-T_{\infty}\right)$ and

$h=h_{w} \frac{T-T_{w}}{T-T_{\infty}}+\sigma_{0} \varepsilon \frac{T^{4}-T_{\infty}^{4}}{T-T_{\infty}}$

and $(z, l)$ strip temperature at position $T(z, l, t)$, where $z$ and $l$ are thickness coordinate and length coordinate, respectively. $\lambda$ is the heat conductivity, $\rho$ the density of strip steel, $c_{p}$ the specific heat capacity, $\varepsilon$ the emission coefficient, $T_{\infty}$ the ambient temperature, $\sigma_{0}$ the Stefan-Boltzmann constant, and $h_{w}$ the convection heat transfer coefficient on the surface of strip.

The radiation boundary condition is only applicable in the air cooling section. The transfer coefficient $h_{w}$ is applicable only in the water cooling section and is calculated as follows:

$h_{w}=\left[2-\left(\frac{N_{c u r}-N_{t}}{10.0+1}\right)^{0.12}\right] \frac{\alpha_{k}}{100}\left(\frac{v}{v_{0}}\right)^{\alpha_{v}}\left(\frac{d}{d_{0}}\right)^{\alpha_{d}}\left(\frac{T}{T_{0}}\right)^{\alpha_{T}}$

where $N_{\text {cur }}$ stands for the current valve position where the moving strip segment arrives. $T, d$ and $v$ are the strip temperature, the thickness and the moving speed, respectively. $v_{0}, d_{0}$ and $T_{0}$ are the values of operating points. $\alpha_{k}, \alpha_{v}, \alpha_{d}$ and $\alpha_{T}$ are proportional coefficient, velocity parameter, thickness parameter and temperature parameter, respectively, which are time-varying parameters.

The thermal conductivity coefficients of the top surface and the bottom surface are defined as

$\lambda(k)=56.43-\left\{0.0363-c\left[v(k)-\left(v\left(t_{h}\right)+\Delta v\right)\right]\right\} T(k)$

where $t_{h}$ is the finish rolling time, $v(k)$ the speed of segment, and $v\left(t_{h}\right)$ the strip speed after the finishing mill. $\Delta v$ and $c$ are velocity increment and velocity coefficient, respectively, which are timevarying parameters.

The thermal diffusivities are defined as

$s_{j}=\left\{\begin{array}{ll}8.65+(5.0-8.65)\left(T_{j}-400\right) / 250 & T_{j} \in[400,650) \\ 5.0+(2.75-5.0)\left(T_{j}-650\right) / 50 & T_{j} \in[650,700) \\ 2.75+(5.25-2.75)\left(T_{j}-700\right) / 100 & T_{j} \in[700,800) \\ 5.25+0.00225\left(T_{j}-800\right) & T_{j} \in[800,1000]\end{array} \quad(j=0,1, \ldots, M)\right.$

\section{References}

Aamodt, A., Plaza, E., 1994. Case-based reasoning: foundational issues, methodological variations, and system approaches. Artif. Intell. Com 7, 39-59.
Aha, D., Breslow, L.A., Munoz-Avila, H., 1999. Conversational case-based reasoning. J. Appl. Intell. 14 (1), 9-32.

Ahn, H., Kim, K.J., 2009. Global optimization of case-based reasoning for breast cytology diagnosis. Expert Syst. Appl. 36 (1), 724-734.

Ahn, H., Kim, K.J., Han, I., 2006. Global optimization of feature weights and the number of neighbors that combine in a case-based reasoning system. Expert Syst. 23 (5), 290-301

Amen, R., Vomacka, P., 2001. Case-based reasoning as a tool for materials selection. Mater. Des. 22 (5), 353-358.

Baskar, S., Subbaraj, P., Rao, M.V.C., 2004. Performance of real-coded genetic algorithms with new genetic operators. J. Inst. Eng. (India) 85, 124-128.

Baskar, S., Subbaraj, P., Rao, M.V.C., Tamilselvi, S., 2003. Genetic algorithms solution to generator maintenance scheduling with modified genetic operators. IEE Proc. Gener. Transm. Distrib. 150 (1), 56-60.

Bedwani, W.A., Ismail, O.M., 2001. Genetic optimization of variable structure PID control system. In: Proceedings of the ACS/IEEE International Conference on Computer Systems and Applications, pp. 27-30.

Bonzano, A., Cunningham, P., Smyth, B., 1997. Using introspective learning to improve retrieval in CBR: a case study in air traffic control. In: Leake, D., Plaza, E. (Eds.), Case-Based Reasoning Research and Development Lecture Notes in Computer Science 1266, Springer-Verlag, New York, pp. 291-302. In: Leake, D. Plaza, E. (Eds.), Case-Based Reasoning Research and Development Lecture Notes in Computer Science 1266, Springer-Verlag, New York, pp. 291-302.

Branting, K., 2001. Acquiring customer preferences from return-set selections In: Proceedings of the Fourth International Conference on Case-based Reasoning 2001(2080), pp. 59-73.

Bryant, S.M., 1997. A case-based reasoning approach to bankruptcy prediction modeling. Intell. Syst. Accounting, Finance Manage. 6 (3), 195-214.

Cercone, N., An, A., Chan, C., 1999. Rule-induction and case-based reasoning: Hybrid architectures appear advantageous. IEEE Trans. Knowl. Data Eng. 11 (1) 166-174.

Chai, T.-Y., Tan, M.-H., Chen, X.-Y., 2002. Intelligent optimization control for laminar cooling. In: Proceedings of the 15th IFAC World Congress, 2002, pp. 181-186.

Chang, P.C., Liu, C.H., Lai, R.K., 2008. A fuzzy case-based reasoning model for sales forecasting in print circuit board industries. Expert Syst. Appl. 34 (3), 2049-2058.

Cheng, Z.-H., Jia, X.-S., Gao, P., Wu, S., Wang, J.-Z., 2008. A framework for intelligent reliability centered maintenance analysis. Reliab. Eng. Syst. Safety 93 (6) 806-814.

Chiu, C.C., Chang, P.C., Chiu, N.H., 2003. A case-based expert support system for due-date assignment in a wafer fabrication factory. J. Intell. Manuf. 14 (3-4) 287-296.

Coello, J.M.A., dos Santos, R.C., 1999. Integrating CBR and heuristic search for learning and reusing solutions in real-time task scheduling, Case-Based Reasoning Research and Development (ICCBR99) LNCS 1650. Springer, Berlin, pp. 89-103.

Coyle, L., Cunningham, P., 2004. Improving recommendation ranking by learning personal feature weights. In: Proceedings of the Seventh European Conference on Case-Based Reasoning 2004(3155), pp. 560-572.

Creecy, R.H., Masand, B.M., Smith, S.J., Waltz, D.J., 1992. Trading MIPS and memory for knowledge engineering. Commun. ACM 35 (8), 48-63.

Cunningham, P., Bonzano, A., Smyth, B., 1997. Using introspective learning to improve retrieval in car: a case study in air traffic control. In: Proceedings of the Second International Conference on Case-Based Reasoning (ICCBR-97). Providence, RI. Springer-Verlag, Berlin, pp. 291-302.

Elhadi, M.T., 2000. Bankruptcy support system: taking advantage of information retrieval and case-based reasoning. Expert Syst. Appl. 18 (3), 215-219.

Fletcher, R., Xu, C., 1987. Hybrid methods for nonlinear least squares. IMA J. Numer. Anal. 7 (3), 371-389.

Goldberg, D.E., 1989. Genetic algorithms in search, Optimization and Machine Learning. Addison-Wesley, Reading, MA.

Gudla, P.K., Ganguli, R., 2005. An automated hybrid genetic-conjugate gradient algorithm for multimodal optimization problems. Appl. Math. Comput. 167 (2), 1457-1474.

Guo, R.-M., 1997. Modeling and simulation of run-out table cooling control using feedforward-feedback and element tracking system. IEEE Trans. Ind. Appl. 33 (2), 304-311.

Guo, R.-M., Hwang, H.T., 1996. Investigation of strip cooling behavior in the run-out section of hot strip mill. J. Mater. Process. Manuf. Sci. 4 (4) 339-351.

Hossain, M.A.,Tokhi, M.O., Chipperfield, A.J., Baxter, M.J., Fonseca, C.M., Dakev, N.V. 1995. Adaptive active vibration control using genetic algorithms. In: IEE Proceedings of the Genetic Algorithms in Engineering Systems: Innovations and Applications, Conference Publication No. 414.

Jarmulak, J., Craw, S., Rowe, R., 2000. Genetic algorithms to optimize CBR retrieval. In: Proceedings of European Workshop on Case-Based Reasoning 2000(1898) pp. 136-147.

Kargupta, H., Smith, R.E., 1991. System identification with evolving polynomial networks. In: Proceedings of the Fourth International Conference on Genetic Algorithm, University of California, San Diego, USA, pp. 370-376.

Kohavi, R., Langley, P., Yun, Y., 1997. The utility of feature weighting in nearest neighbor algorithms. In: Poster Paper of the Ninth European Conference on Machine Learning (ECML-97), Prague.

Kolodner, J.L., 1993. Case-based Reasoning. Morgan Kaufmann Publisher, San Francisco, USA 
Kristinsson, K., Dumont, G., 1992. System identification and control using genetic algorithms. IEEE Trans. Syst., Man and Cybernet. 22 (5), 1033-1046.

Kwong, C.K., Tam, S.M., 2002. Case-based reasoning approach to concurrent design of low power transformers. J. Mater. Process. Technol. 128 (1-3), 136-141.

Li, S.T., Ho, H.F., 2009. Predicting financial activity with evolutionary fuzzy casebased reasoning. Expert Syst. Appl. 36 (1), 411-422.

Liu, J.-F., Cai, F.-L., Shuai, Z.-W., 2009. Research on management scheduling based on simulated annealing and gentic algorithm. Time Space Software 25 (9-3), 199-201.

Makeyev, O., Sazonov, E., Moklyachuk, M., Meyer, P.L., 2010. Hybrid evolutionary algorithm for microscrew thread parameter estimation. Eng. Appl. Artif. Intel. 23 (4), 446-452.

Mejasson, P., Petridis, M., Knight, B., Soper, A., Norman, P., 2001. Intelligent design assistant (IDA): a case base reasoning system for material and design. Mater. Des. 22 (3), 163-170.

Mileman, T., Knight, B., Petridis, M., Cowell, D., Ewer, J., 2002. Case-based retrieval of 3-dimensional shapes for the design of metal castings. J. Intell. Manuf. 13 (1), 39-45.

Nikolaychuk, O.A., Yurin, A.Y., 2008. Computer-aided identification of mechanical system's technical state with the aid of case-based reasoning. Expert Syst. Appl. 34 (1), 635-642.

Pehlke, R.D., Jeyerajan, A., Wada, H., 1982. Summary of Thermal Properties for Casting Alloys and Mold Materials. Department Materials and Metallurgical Engineering, University of Michigan, Ann Arbor.

Pian, J.-X., Chai, T.-Y., 2008. Hybrid intelligent parameter identification of the laminar cooling process. Control Theory Appl. 25 (5), 933-937.

Press, W., Teukolsky, S., Vetterling, W., Flannery, B., 2007. Numerical Recipes, 3rd Edition Cambridge University Press, New-York The Art of Scientific Computing.

Ricci, F., Avesani, P., 1995. Learning a local similarity metric for case-based reasoning. Case-Based Reasoning Research and Development (ICCBR95) LNCS 1010. Springer, Berlin, pp. 301-312.

Rivard, H., Fenves, S.J., 2000. SEED-Config: a case-based reasoning system for conceptual building design. Artif. Intell. Eng. Des., Anal. Manuf. 14 (5), 415-430.

Serajzadeh, S., 2004. Modeling of temperature history and phase transformations during cooling steel. J. Mater. Process. Technol. 146 (3), 311-317.

Sha, Q.-Y., Li, G.-Y., Qiao, L.-F., Yan, P.-Y., 2007. Effect of cooling rate and coiling temperature on precipitate in ferrite of a $\mathrm{Nb}-\mathrm{V}-\mathrm{Ti}$ microalloyed strip steel. J. Iron Steel Res. 14 (5), 316-319.

Shin, K., Han, I., 1999. Case-based reasoning supported by genetic algorithms for corporate bond rating. Expert Syst. Appl. 16 (2), 85-95.

Soderstrom, T., Stoica, P., 1984. System Identification. Prentice Hall, pp. 442-451.
Stahl, A., Gabel, T., 2003. Using evolution programs to learn local similarity measures. In: Proceedings of the Fifth International Conference on Case-based Reasoning 2003(2689), pp. 537-551.

Subbaraj, P., Rengaraj, R., Salivahanan, S., 2011. Enhancement of Self-adaptive realcoded genetic algorithm using Taguchi method for economic dispatch problem. Appl. Soft Comput. 11 (1), 83-92.

Suh, M.S., Jhee, W.C., Ko, Y.K., Lee, A., 1998. A case-based expert system approach for quality design. Expert Syst. Appl. 15 (2), 181-190.

Sun, J., Hui, X.-F., 2006. Financial distress prediction based on similarity weighted voting CBR. Adv. Data Mining Appl. 4093 (2006), 947-958.

Sun, J., Li, H., 2009. Financial distress early warning based on group decision making. Comput. Operat. Res. 36 (3), 885-906.

Tan, M.-H., Chai, T.-Y., 2005. Modeling of the laminar cooling process with casebased reasoning. Control Theory. Appl. 22 (2), 248-253.

Tang, Z.-Y., Ding, H., Du, L.-X., Zhang, X., 2007. Effect of thermo mechanical processing on microstructures of trip steel. J. Iron Steel Res. 14 (2), 56-60.

Tavakolpour, A.R., Mat Darus, I.Z., Tokhi, O., Mailah, M., 2010. Genetic algorithmbased identification of transfer function parameters for a rectangular flexible plate system. Eng. Appl. Artif. Intell. 23 (8), 1388-1397.

Tjoa, I.B., Biegler, L.T., 1991. Simultaneous solution and optimization strategies for parameter estimation of differential-algebraic equation systems. Ind. Eng. Chem. Res. 30 (2), 376-385.

Watson, I., Gardingen, D.A., 1999. Distributed case-based reasoning application for engineering sales support. In: Proceedings of 16th International Joint Conference on Artificial Intelligence (IJCAI-99), San Francisco, CA: Morgen Kaufmann Publishers, vol. 1, pp. 600-605.

Wettschereck, D., Aha, D., 1995. Weighting features. In: Proceedings of the First International Conference on Case-based Reasoning, 1995(1010), pp. 347-358.

Wilke, W., Lenz, M., Wess, S., 1998. Intelligent sales support with CBR, Case-based Reasoning Technology, LNCS 1400. Springer, Berlin, pp. 91-113.

Xiong, N., 2011. Learning fuzzy rules for similarity assessment in case-based reasoning. Expert Syst. Appl. 38 (9), 10780-10786.

Xiong, N., Funk, P., 2006. Building similarity metrics reflecting utility in case-based reasoning. J. Intell. Fuzzy Syst. 17 (4), 407-416.

Yu, W.D., Liu, Y.C., 2006. Hybridization of CBR and numeric soft computing techniques for mining of scarce construction databases. Automat. Construct. 15 (1), 33-46.

Zheng, Y., Li, S.-Y., Wang, X.-B., 2009. Distributed model predictive control for plant-wide hot-rolled strip laminar cooling process. J. Process. Control 19 (9), 1427-1437. 